\title{
Introducing Transmission Electron Microscopy to High School Students
}

\author{
J. R. McBride*
}

* Department of Chemistry, Vanderbilt University, 7300 Stevenson Center, Nashville, TN 37221

As nanoscience and technology moves from strictly a university setting to a growing industry, it is essential to introduce this field to high school and college students. Not only are there new jobs associated with this industry, but also there is a growing presence of nanotechnology behind the products we buy and use every day. In addition to introducing students to nano-phenomena, they must be able to visualize something that, to them, is simply 'really really small'. This is where electron microscopy can shine. Between scanning electron microscopy (SEM) and transmission electron microscopy (TEM), SEM can be the simplest to explain with the formation of the image similar to how we see objects everyday. However, to image objects with dimension on the order of nanometers requires the use of TEM.

Vanderbilt University and the Vanderbilt Institute of Nanoscale Science and Engineering (VINSE) host two groups of high school students each summer. As part of their introduction to nanotechnology, a 10-20 minute demonstration of the $200 \mathrm{kV}$ Phillips CM20 High Resolution (HR) TEM is included. During this demonstration the students are first introduced to the concepts of why electrons are used, spatial resolution, the generation of the electron beam, how the beam is shaped, and constructive and destructive interference in regards to phase-contrast imaging. This introduction is brief, involving a presentation that includes imagery to bridge the gap between the student's rudimentary understanding and the often abstract workings of the microscope.

The key part of any microscopy demonstration to high school students is a visually compelling sample. At Vanderbilt, we use 6-8 nm colloidal CdSe nanocrystals (quantum dots) deposited onto an ultrathin carbon coated TEM grid as the sample. The size-dependent optical properties of the quantum dots are a very clear demonstration of a nano-phenomenon (Figure 1). Additionally, the nanocrystals' hexagonal crystal structure can be readily imaged and compared to a ball ' $\mathrm{n}$ stick model that the students are allowed to handle. Figure 2 contains a pair of HRTEM images of CdSe nanocrystals exhibiting lattice fringes. The students are encouraged to rotate the nanocrystal model to match what they see in the TEM. Lastly, the students are given a chance to 'drive', where they can move to new areas of the sample and try to focus the image. 


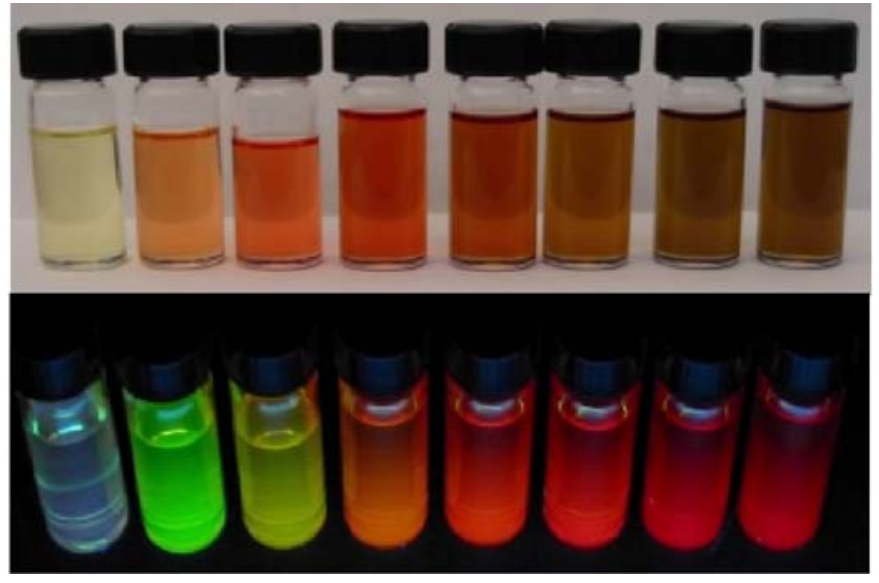

FIG. 1. A size series of CdSe nanocrystals ranging from 2 to $8 \mathrm{~nm}$ in diameter (left to right). As the diameter of the nanocrystal increases, the absorption (top) and the emission (bottom) shifts to longer wavelengths.
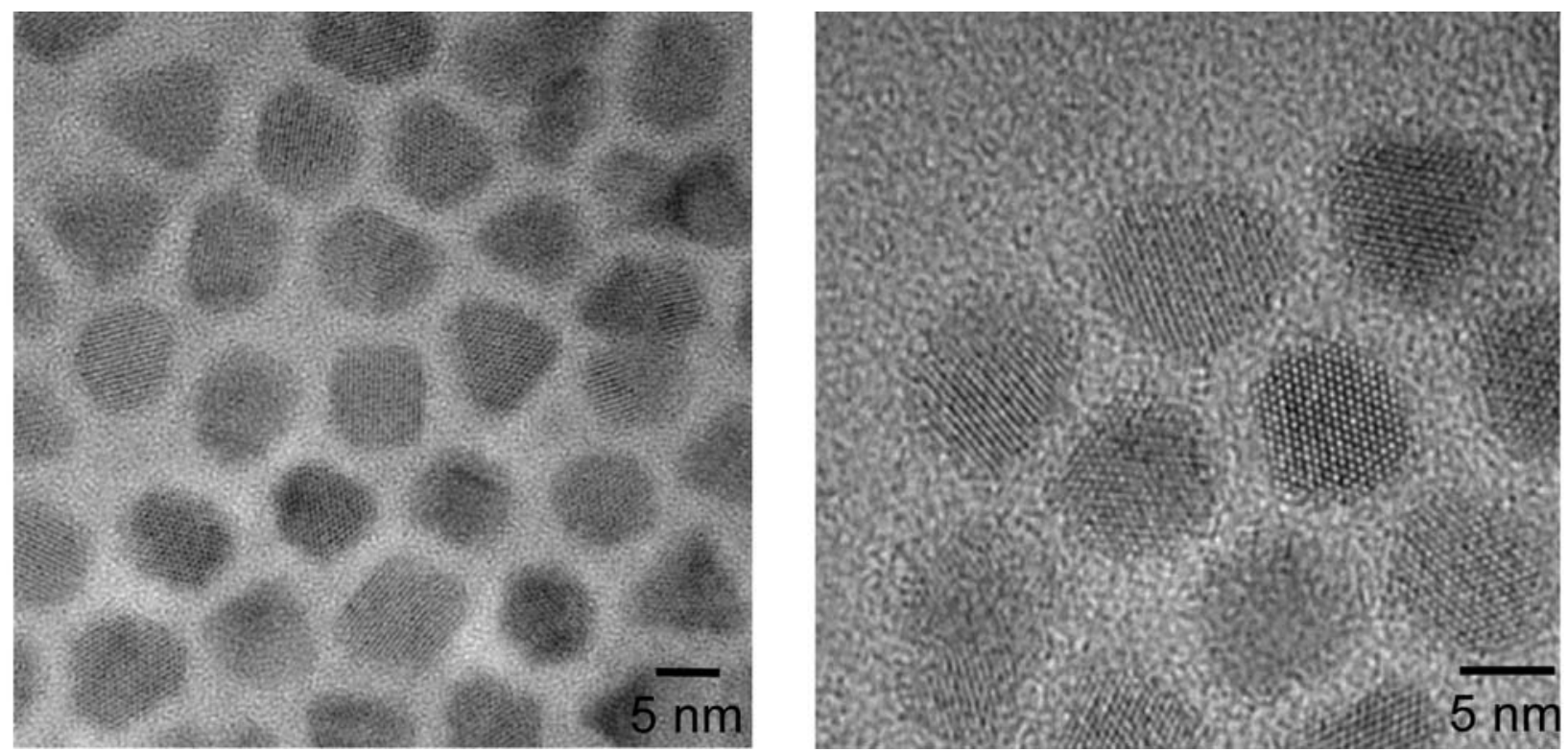

FIG. 2. HRTEM images of CdSe nanocrystals on an ultrathin carbon coated TEM grid. The nanocrystals exhibit different fringe patterns and shapes depending on their orientation relative to the electron beam. 